\title{
Télescope
}

Revue d'analyse comparée en administration publique

\section{L'explosion de l'audit dans le secteur public : le risque d'une illusion de contrôle}

\section{Marie-Soleil Tremblay et Bertrand Malsch}

Volume 18, numéro 3, automne 2012

URI : https://id.erudit.org/iderudit/1013772ar

DOI : https://doi.org/10.7202/1013772ar

Aller au sommaire du numéro

Éditeur(s)

L’Observatoire de l'administration publique

ISSN

1203-3294 (imprimé)

1929-3348 (numérique)

Découvrir la revue

Citer ce document

Tremblay, M.-S. \& Malsch, B. (2012). L'explosion de l'audit dans le secteur public : le risque d'une illusion de contrôle. Télescope, 18(3), 1-7.

https://doi.org/10.7202/1013772ar
Résumé de l'article

La gestion des administrations publiques fondée sur les résultats et la transparence est employée fréquemment par les politiciens et les fonctionnaires comme une façon de promouvoir l'efficacité et les idéaux de réforme destinés à transformer les organisations du secteur public (Hood, 1995, 1990). Le présent essai porte un regard critique sur l'explosion des pratiques de vérification au sein des organisations du secteur public afin de mieux comprendre comment ces changements ont été introduits dans les organisations publiques, de s'interroger sur leurs possibles effets involontaires et de souligner les risques d'une illusion de contrôle. 


\title{
L'EXPLOSION DE L'AUDIT DANS LE SECTEUR PUBLIC : LE RISQUE D'UNE ILLUSION DE CONTRÔLE
}

\author{
Par Marie-Soleil Tremblay, Professeure, École nationale d'administration publique • \\ marie-soleil.tremblay@enap.ca \\ Et Bertrand Malsch, Professeur adjoint, HEC Montréal • bertrand.malsch@hec.ca \\ Traduit de l'anglais
}

\begin{abstract}
RÉSUMÉ La gestion des administrations publiques fondée sur les résultats et la transparence est employée fréquemment par les politiciens et les fonctionnaires comme une façon de promouvoir l'efficacité et les idéaux de réforme destinés à transformer les organisations du secteur public (Hood, 1995, 1990). Le présent essai porte un regard critique sur l'explosion des pratiques de vérification au sein des organisations du secteur public afin de mieux comprendre comment ces changements ont été introduits dans les organisations publiques, de s'interroger sur leurs possibles effets involontaires et de souligner les risques d'une illusion de contrôle.
\end{abstract}

\begin{abstract}
Politicians and public servants have turned to managing by results and increased transparency, rather than policy options, as a way to promote efficiency and ideals of reformability - and ultimately to transform public sector organizations, as has been articulated by Hood $(1995,1990)$. This essay is therefore concerned with the explosion of audits in public sector organizations with a view to better understanding how change has been introduced within the public sector, questioning its possible unintended effects and highlighting the risks of an illusion of control.
\end{abstract}

Pour citer cet article : Tremblay, M.-S. et B. Malsch (2012). « L'explosion de l'audit dans le secteur public : le risque d'une illusion de contrôle », Télescope, vol. 18, n³, p. 1-7.

\begin{abstract}
À la suite des nombreux scandales financiers de la dernière décennie, certains ont prétendu que les systèmes de contrôle des organisations publiques pourraient se révéler défectueux et que des réformes semblables à la loi SarbanesOxley ${ }^{1}$ seraient nécessaires au sein des organisations gouvernementales et sans but lucratif pour éviter des désastres comparables (Brown, 2005; Jackson et Fogarty, 2005; Roberts et Candreva, 2006). Les conseils d'administration des sociétés d'État ont ainsi vu leurs responsabilités s'accroître de façon importante en matière de gestion des risques et de contrôle interne, l'objectif étant une amélioration de l'efficacité et de l'imputabilité (Free et Radcliffe, 2009; Gendron, Cooper et Townley, 2007). L'implantation massive des pratiques de vérification dans le secteur public, comme moyen de contrôle, a été inspirée très directement des méthodes en cours dans les entreprises du secteur privé. Cependant, l'importation de telles pratiques
\end{abstract}

1 La loi Sarbanes-Oxley (Sarbanes Oxley Act), ou loi SOX, est une loi fédérale américaine édictée en 2002 qui fixe de nouveaux standards pour tous les conseils d'administration des sociétés ouvertes et pour toutes les directions de firmes de gestion et des cabinets comptables des États-Unis. 
et techniques a généré des débats critiques sur leur nécessité, leur pertinence et leur valeur ajoutée (Clatworthy, Mellett et Peel, 2000; Vermeer, Raghunandan et Forgione, 2006).

\section{- LES PROGRAMMES ET LES TECHNOLOGIES DE VÉRIFICATION}

Miller et Rose (2008) prétendent que l'exercice du gouvernement implique à la fois l'usage de programmes et de technologies. D'une part, les programmes sont des idéaux qui représentent et problématisent des activités de telle façon qu'elles deviennent " gouvernables ", c'est-à-dire contrôlables. Ces programmes prennent généralement la forme de rapports, d'articles ou de livres blancs. D'autre part, les technologies constituent les divers instruments et outils qui rendent les programmes opérationnels. Cependant, les frontières entre les programmes et les technologies sont parfois floues. Les idées et les concepts qui façonnent les technologies et les pratiques sont souvent attachés à des programmes plus vastes (Miller et Rose, 2008; Ogden, 1997; Power, 1997; Preston, Cooper et Coombs, 1992). La vérification, par exemple, peut être vue comme programmatique ou alors comme une technologie tributaire de certains programmes.

L'abstraction des idées est l'une des caractéristiques majeures de la société moderne (Giddens, 1991 et 1990). Dans notre univers globalisé, les idées, les modèles et les normes transgressent les barrières du temps et de l'espace; les discours émergent dans un contexte local pour se transformer à un niveau global en formes abstraites prêtes à être réinsérées dans d'autres contextes (Czarniawska et Sevón, 2005). L'adoption d'idées, ou dans ce cas particulier le développement des pratiques de vérification au sein du secteur public, dépend ainsi en grande partie de leur théorisation, c'est-à-dire de leur transformation en concepts abstraits présentant l'idée comme une solution à un problème précis (Greenwood, Suddaby et Hinings, 2002). En d'autres termes, le processus de théorisation constitue une étape cruciale qui permet à une idée de se répandre dans la société. Il implique la définition des problèmes que la réglementation proposée entend résoudre de même que la justification du traitement proposé (Tolbert et Zucker, 1996). Comme le font remarquer Strang et Meyer (1993), la théorisation joue également un rôle très important pour traduire et légitimer les idées complexes en des formats facilement compréhensibles et convaincants.

L'adoption des pratiques de vérification dans le secteur public canadien s'est ainsi construite à partir d'un programme centré et théorisé autour des notions d'efficacité et de modernisation. En agissant de la sorte, les autorités législatives du Canada et des provinces n'ont fait qu'emboîter le pas à de nombreux autres gouvernements dans l'hémisphère occidental qui ont su se faire élire en s'engageant à " moderniser " leur secteur public en faisant la promotion des pratiques du secteur privé (Broadbent et Guthrie, 2008; English et Skærbæk, 2007; Hood et Peters, 2004; Lapsley, 2008 et 2009). Ainsi, la gestion par résultats, plutôt que par activités, a entraîné une forte demande de vérification dans le secteur public comme un moyen indispensable de responsabilisation et une garantie de qualité. Par conséquent, le refus de se soumettre à une vérification, ou un manque de coopération, est 
désormais considéré comme une tentative grave de dissimuler un secret ou des activités frauduleuses (Power, 1994).

Fer de lance de la modernisation du secteur public, la vérification est devenue un slogan essentiel dans la bouche de ceux qui souhaitent accroître la responsabilisation des administrateurs (Roe, 1994). Sur la scène internationale, l'OCDE offre depuis 1999 des standards à caractère non obligatoire sur la façon de gérer des entreprises et en a produit une version révisée en 2004 (OCDE, 2004). Dans ces documents, les systèmes de gouvernance des entreprises privées et leurs technologies de vérification sont présentés comme des outils performants pour accroître la confiance dans les marchés financiers et réduire le coût du capital des entreprises. En se basant sur ces standards, des " experts " du monde entier ont ainsi encouragé le déploiement de ces systèmes et de ces technologies, en particulier dans le secteur public. En 2005, l'OCDE a publié les Lignes directrices de l'OCDE sur le gouvernement d'entreprise des entreprises publiques (OCDE, 2005), dont l'objectif était d'" assister » les gouvernements dans le défi représenté par l'évaluation et l'amélioration de leur manière d'exercer le contrôle de ces entreprises :

[L] es entreprises publiques posent un certain nombre de problèmes spécifiques de gouvernement d'entreprise. L'une de ces difficultés tient au fait que les entreprises publiques peuvent souffrir au moins autant d'ingérences politiques directes indues que de la passivité totale ou de la distance de l'État actionnaire. On assiste quelquefois aussi à une dilution des responsabilités. Les entreprises publiques sont souvent à l'abri de deux menaces qui sont essentielles au contrôle de la gestion dans le secteur privé, à savoir la menace d'une [offre publique d'achat] et celle d'une faillite (OCDE, 2005, p. 3).

Inspirées par les pratiques du secteur privé, les lignes directrices dont l'OCDE fait la promotion font l'apologie des fonctions d'audit interne supervisées par des comités de vérification indépendants, des vérificateurs externes indépendants, de la publication des facteurs de risque et des mesures destinées à gérer ces risques. Ces idéaux ont trouvé des échos divers tels que la publication de livres blancs sur les bonnes pratiques (par exemple le rapport Saucier - Canadian Institute of Chartered Accountants, 2001) et l'élaboration de standards " innovants " en matière de réglementations pour les sociétés cotées en Bourse.

\section{- LA VÉRIFICATION DANS LE SECTEUR PUBLIC : UNE ILLUSION DE CONTRÔLE?}

Comme la budgétisation et la comptabilité d'exercice (Carlin, 2005) ou les mesures de rendement dans le secteur public (Townley, Cooper et Oakes, 2003), les promesses liées à la vérification ne semblent pas avoir été tenues et semblent avoir été avancées essentiellement sur des bases idéologiques. L'analyse des débats publics montre que l'adoption de l'idéal de vérification par le législateur s'est faite à partir d'une appréciation superficielle de la réalité, sans analyses empiriques sérieuses. Comme le suggèrent Flottes et Gendron (2010), la superficialité est l'une des principales caractéristiques du processus par lequel les lois et les règlementations ont actuellement tendance à s'établir dans la société contemporaine. 
Clarke et Dean (2007) se montrent très sceptiques face aux diverses procédures de gouvernance et de vérification mises sur pied à la suite des échecs des entreprises privées, procédures destinées, selon eux, à sauver les apparences (c'est-à-dire à légitimer) plutôt qu'à rectifier les problèmes de fond. Comment la gestion des risques, les procédures de contrôle interne, l'augmentation des audits internes et l'étalonnage peuvent-ils empêcher les scandales locaux de se produire? Comment devrait-on déployer des technologies afin de s'assurer de leurs bienfaits sur la gouvernance des sociétés? Power (2009) soutient que les technologies de vérification communiquent aux différentes parties prenantes une fausse impression de sécurité. Les experts en vérification et en gestion de risque continuent toutefois de vendre les technologies associées à ces programmes comme si la société du risque dans laquelle nous vivons était mesurable, quantifiable et contrôlable (Gabe, 1995).

En théorie, l'objectivité et l'indépendance du vérificateur ainsi que la rigueur quasi scientifique des processus d'audit sont essentiels à l'État et, de manière plus générale, à la confiance que témoigne l'opinion publique envers le mécanisme de surveillance. Cependant, comme le remarque Pentland :

Chaque vérification est en quelque sorte une expérience sans contrôle et il n'existe pas de manière de savoir ce qui se serait passé si une vérification n'avait pas été faite. Il n'est pas étonnant que les vérifications soient obscures sur le plan épistémologique - les vérificateurs ont adopté la rhétorique de la méthode scientifique sans véritablement parvenir à en adopter beaucoup de substance (Pentland, 2000, p. 311).

En somme, quelles que soient l'illusion de contrôle en cours et son apparence de puissance, le rapport du vérificateur n'est pas basé sur des certitudes mathématiques. Les vérifications sont fondamentalement subjectives, elles sont matière à interprétation (Francis, 1994), imprévisibles (Morin, 2002) et incontrôlables.

\section{- DISCUSSION ET CONCLUSION}

Les programmes et les technologies de vérification sont parvenus à voyager à travers le monde avec succès, davantage en raison de leur rhétorique sous-jacente qu'en raison de leur efficacité prouvée à transformer positivement le fonctionnement du secteur public (Parker et Gould, 1999). De tels programmes ne se déplacent pas sans aide; ils sont véhiculés par différentes rationalités puissantes. La difficulté consiste cependant à distinguer les caractères économiques, politiques, sociaux ou comptables de ces différents types de rationalités «impliqués dans la construction de ce qui est désirable" (Hopwood, 1984, p. 171).

À la lumière de cet essai et des études précédentes sur le sujet (comme Lapsley et Pong, 2000), il apparaît que les idées et les réformes de gouvernance publique seraient mises en marché à l'aide d'arguments très simplistes. Il arrive fréquemment que les défis et la nécessité de prendre en compte la complexité du monde soient évités, ce qui soulève la question suivante : comment rendre visible ce qui est complexe et ce qui est problématique? Ironiquement, démontrer la complexité est une tâche complexe. Non seulement parce que la démarche requiert des 
connaissances étendues et une réflexion approfondie, mais également parce que la plupart des citoyens s'attendent à ce que les politiciens fournissent des certitudes sous la forme de solutions simples et faciles.

Des recherches plus avancées pourraient se pencher sur les conséquences volontaires et involontaires liées aux réformes de l'audit dans le secteur public. Les experts de la recherche appliquée pourraient suivre des vérificateurs et chercher à améliorer leurs technologies, en se concentrant sur les moyens plutôt que sur les résultats. D'autres, plus critiques ou remettant en doute les avantages de ces technologies, préféreront mettre en lumière le réseau des intérêts sans cesse en expansion des connexions d'arrière-scène qui soutiennent l'évolution des technologies de la vérification.

Comme le souligne Power (1997, p. 144), ce serait faire fausse route de conclure cet essai en affirmant qu'il est souhaitable de diminuer les pratiques de vérification dans le secteur public. Plutôt que de brûler ce qui a été adoré, il faut au contraire chercher à donner sa juste place à la vérification et à la considérer pour ce qu'elle est, c'est-à-dire une technique à la fois sérieuse et faillible qui permet dans certaines circonstances d'améliorer l'efficacité des services rendus au citoyen.

\section{BIBLIOGRAPHIE}

Broadbent, J. et J. Guthrie (2008). "Public Sector to Public Services: 20 Years of "Contextual" Accounting Research ", Accounting, Auditing \& Accountability Journal, vol. 21, n² 2, p. 129-169.

Brown, R. E. (2005). "Enron/Andersen: Crisis in U.S. Accounting and Lessons for Government ", Public Budgeting \& Finance, vol. 25, n³ 3, p. 20-32.

Canadian Institute of Chartered Accountants (2001). Beyond Compliance: Building a Governance Culture, www.ecgi.org/codes/documents/beyond_compliance.pdf (page consultée en juillet 2012).

Carlin, T. M. (2005). " Debating the Impact of Accrual Accounting and Reporting in the Public Sector ", Financial Accountability \& Management, vol. 21, n 3, p. 309-336.

Clarke, F. et G. Dean (2007). Indecent Disclosure: Gilding the Corporate Lily, Cambridge, Cambridge University Press.

Clatworthy, M., H. Mellett et M. Peel (2000). " Corporate Governance under "New Public Management": An Exemplification ", Corporate Governance: An International Review, vol. $8, n^{\circ} 2$, p. 166-176.

Czarniawska, B. et G. Sevón (2005). " Translation is a Vehicle, Imitation its Motor and Fashion Sits at the Wheel », dans B. Czarniawska et G. Sevón (dir.), Global Ideas: How Ideas, Objects and Practices Travel in the Global Economy, Malmö, Liber \& Copenhagen Business School Press.

English, L. et P. Skærbæk (2007). "Performance Auditing and the Modernization of the Public Sector ", Financial Accountability and Management, vol. 23, n 3, p. 239-241. 
Flottes, M. et Y. Gendron (2010). "Sarbanes-Oxley et l'indépendance des auditeurs : mouvance réglementaire et jeux de vérité ", Comptabilité - Contrôle - Audit, vol. 16, $\mathrm{n}^{\circ} 2$, p. $7-29$.

Francis, J. R. (1994). "Auditing, Hermeneutics, and Subjectivity ", Accounting, Organizations and Society, vol. 19, n 3, p. 235-269.

Free, C. et V. Radcliffe (2009). « Accountability in Crisis: The Sponsorship Scandal and the Office of the Comptroller General in Canada ", Journal of Business Ethics, vol. 84, $\mathrm{n}^{\circ} 2$, p. 189-208.

Gabe, J. (1995). " Health, Medicine and Risk: The Need for a Social Approach », dans J. Gabe (dir.), Medicine, Health and Risk: Sociological Approaches, Oxford, Blackwell.

Gendron, Y., D. J. Cooper et B. Townley (2007). « The Construction of Auditing Expertise in Measuring Government Performance ", Accounting, Organizations \& Society, vol. 32, $\mathrm{n}^{\circ} 1 / 2$, p. 105-133.

Giddens, A. (1991). Modernity and Self-identity: Self and Society in the Late Modern Age, Stanford, Stanford University Press.

Giddens, A. (1990). The Consequences of Modernity, Stanford, Stanford University Press.

Greenwood, R., R. Suddaby et C. R. Hinings (2002). " Theorizing Change: The Role of Professional Associations in the Transformation of Institutionalized Fields ", The Academy of Management Journal, vol. 45, $n^{\circ}$ 1, p. 58-80.

Hood, C. (2007). « What Happens When Transparency Meets Blame-avoidance? ", Public Management Review, vol. 9, $\mathrm{n}^{\circ}$ 2, p. 191-210.

Hood, C. (1995). "The "New Public Management" in the 1980s: Variations on a Theme ", Accounting, Organizations \& Society, vol. 20, n² 2/3, p. 93-109.

Hood, C. (1990). "A Public Management for All Seasons ", Public Administration, vol. 69, p. 3-19.

Hood, C. et G. Peters (2004). "The Middle Aging of New Public Management: Into the Age of Paradox? ", Journal of Public Administration Research \& Theory, vol. 14, $\mathrm{n}^{\circ} 3$, p. 267-282.

Hopwood, A. G. (1984). "Accounting and the Pursuit of Efficiency ", dans A. G. Hopwood et C. Tonkins (dir.), Issues in Public Sector Accounting, Oxford, Philip Allan.

Jackson, P. M. et T. E. Fogarty (2005). Sarbanes-Oxley for Nonprofits: A Guide to Gaining Competitive Advantage, Hoboken, John Wiley \& Sons, Inc.

Lapsley, I. (2009). " New Public Management: The Cruellest Invention of the Human Spirit? ", Abacus, vol. 45, n 1, p. 1-21.

Lapsley, I. (2008). "The NPM Agenda: Back to the Future ", Financial Accountability \& Management, vol. 24, $\mathrm{n}^{\circ} 1$, p. 77-96.

Lapsley, I. et C. K. M. Pong (2000). « Modernization Versus Problematization: Value-formoney Audit in Public Services ", European Accounting Review, vol. 9, n 4, p. 541-567.

Miller, P. et N. Rose (2008). Governing the Present: Administering Economic, Social and Personal Life, Cambridge, Polity Press.

Morin, D. (2002). "Influence of Value for Money Audit on Public Administrations: Looking Beyond Appearances ", Financial Accountability \& Management, vol. 17, n² 2, p. 99-117. 
Ogden, S. (1997). " Accounting for Organizational Performance: The Construction of the Customer in the Privatized Water Industry ", Accounting, Organizations and Society, vol. $22, n^{\circ} 6$, p. 529-556.

OCDE (2005). Lignes directrices de l'OCDE sur le gouvernement d'entreprise des entreprises publiques, www.oecd.org/daf/corporateaffairs/soe/guidelines (page consultée en mars 2009).

OCDE (2004). OECD Principles of Corporate Governance, www.oecd.org/dataoecd/32/18/ 31557724.pdf (page consultée en mars 2009).

Parker, L. et G. Gould (1999). " Changing Public Sector Accountability: Critiquing New Directions ", Accounting Forum, vol. 23, n 2, p. 109-135.

Pentland, B. T. (2000). "Will Auditors Take Over the World? Program, Technique and the Verification of Everything ", Accounting, Organizations and Society, vol. 25, n 3, p. $307-312$.

Power, M. (2009). " The Risk Management of Nothing ", Accounting, Organizations and Society, vol. 34, n 6-7, p. 849-855.

Power, M. (2004). The Risk Management of Everything: Rethinking the Politics of Uncertainty, Londres, Demos.

Power, M. (1997). The Audit Society: Rituals of Verification, Oxford, Oxford University Press.

Power, M. (1994). The Audit Explosion, Londres, Demos.

Preston, A. M., D. J. Cooper et R. W. Coombs (1992). "Fabricating Budgets: A Study of the Production of Management Budgeting in the National Health Service ", Accounting, Organizations \& Society, vol. 17, n 6, p. 561-593.

Roberts, N. et P. J. Candreva (2006). "Controlling Internal Controls ", Public Administration Review, vol. 66, n 3, p. 463-465.

Roe, E. (1994). Narrative Policy Analysis: Theory and Practice, Durham, Duke University Press.

Strang, D. et J. W. Meyer (1993). "Institutional Conditions for Diffusion ", Theory and Society, vol. 22, n 4 , p. 487-511.

Tolbert, P. S. et L. G. Zucker (1996). "Institutionalization of Institutional Theory ", dans S. Clegg, C. Hardy et W. Nord (dir.), Handbook of Organization Studies, Londres, Sage.

Townley, B., D. J. Cooper et L. Oakes (2003). "Performance Measures and the Rationalization of Organizations ", Organization Studies, vol. 24, n 7, p. 1045-1071.

Tremblay, M.-S. et Y. Gendron (2011). " Governance Prescriptions Under Trial: On the Interplay Between the Logics of Resistance and Compliance in Audit Committees ", Critical Perspectives on Accounting, vol. 22, n 3, p. 259-272.

Vermeer, T. K., K. Raghunandan et D. A. Forgione (2006). "The Composition of Non Profit Audit Committees ", Accounting Horizons, vol. 20, n 1, p. 75-90. 